\title{
Evaluation study of hypernuclei production at NICA/MPD
}

\author{
Mariya Ilieva $^{1,2, *}$, Alexander Zinchenko ${ }^{1}$, and Vadim Kolesnikov ${ }^{1}$ \\ ${ }^{1}$ VBLHEP, Joint Institute for Nuclear Research, 141980 Dubna, Moscow region, Russia \\ ${ }^{2}$ Plovdiv University "Paisii Hilendarski", Plovdiv, Bulgaria
}

\begin{abstract}
The main goal of the NICA/MPD facility is a study of the properties of hot and dense baryonic matter. One of the possible ways of doing this is to study strangeness production in nuclear collisions. The enhanced production of strange particles in heavy-ion collisions is considered to be a signal for the quark-gluon plasma formation. In this paper the MPD detector abilities to measure different hypernuclei species in $\mathrm{Au}+\mathrm{Au}$ collisions at NICA energy region are presented.
\end{abstract}

\section{Introduction}

The main purpose of NICA (new collider complex at the Joint Institute for Nuclear Research) is to study the behaviour of nuclear matter under extreme conditions [1]. The energy range of the NICA collider $(4<\sqrt{s}<11 \mathrm{~A} \mathrm{GeV})$ allows an experimental exploration of fundamental QCD properties that are sensitive to chiral symmetry and confinement phenomena [2]. Our experimental research plan is to perform a detailed energy scan with ion beams from protons to gold nuclei addressing the following objectives: (1) strangeness production, (2) in-medium properties of vector mesons, (3) event-by-event fluctuations and (4) correlations. The measurements will be performed with the MultiPurpose Detector (MPD) capable of detecting both the hadronic ( $\pi, K, p, \Lambda, \Xi, \Omega)$ and non-hadronic $(e, \gamma)$ probes.

The abundance of strange particles in relativistic heavy-ion collisions offers a unique possibility to study exotic nuclear objects with strangeness - hypernuclei [3]. The new precise experimental data on the total yields, rapidity, transverse momentum, and azimuthal angle distributions of hyperons, provided by the MPD experiment at NICA, will give the opportunity to better understand the dynamics of hot and dense hadronic matter.

NICA energy range covers the region of the maximal baryon density, where the production rates of nuclear clusters with strangeness are predicted to be enhanced considerably: as many as $3 \cdot 10^{2}$ of ${ }_{\Lambda}^{3} \mathrm{H}$ and $1 \cdot 10^{-5}$ of ${ }_{\Lambda \Lambda}^{5} \mathrm{He}$ per unit of rapidity are expected in a central $\mathrm{Au}+\mathrm{Au}$ collision at $\sqrt{s}=5 \mathrm{~A} \mathrm{GeV}$ [4]. With a typical event rate of $6 \mathrm{kHz}$ for the design NICA luminosity of $10^{27} \mathrm{~cm}^{-2} \mathrm{c}^{-1}$, a detailed study of the production mechanism of single hypernuclei as well as an observation of double hypernuclei at NICA seems feasible.

\section{MPD Detector}

The present analysis is based on the MPD Stage I configuration (Fig. 1), which includes the main tracker Time Projection Chamber (TPC) supplemented by the Time-Of-Flight system

\footnotetext{
*e-mail: maiailieva@mail.bg
} 


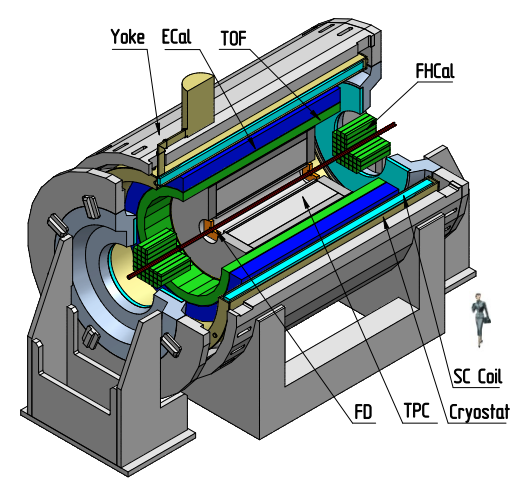

Figure 1. Geometry of MPD Stage I

(TOF) for precise momentum measurements and particle identification. The Forward Detector (FD) will serve as a start trigger, Electromagnetic Calorimeter (ECAL) for measurements of electromagnetic showers, Forward Hadron Calorimeter (FHCal) for determination of the collision centrality and the orientation of the reaction plane for collective flow studies. The detectors cover the mid-rapidity region $|\eta|<1.3$. The detailed description of the MPD geometry can be found in [2].

\section{Data set}

The event sample for the present study has been produced with the DCM-QGSM (Dubna Cascade Model - Quark-Gluon String Model) generator [5, 7]. The choice of the DCMQGSM event generator was motivated by the fact that it can produce nuclear clusters via the coalescence mechanism and describe hypernuclei yield in NICA energy region. The DCMQGSM generator is based on the Monte Carlo solution of a set of the Boltzmann-UehlingUhlenbeck relativistic kinetic equations with the collision terms, including cascade-cascade interactions. For particle energies below $1 \mathrm{GeV}$ it considers only nucleons, pions and deltas. The model includes a proper description of pion and baryon dynamics for particle production and absorption processes. At energies higher than about $5 \mathrm{GeV}$, the Quark-Gluon String Model is used to describe elementary hadron collisions.

DCM-QGSM model predictions for cluster formation have been compared with experimental data $[8,9]$. The model was used to predict cluster production over a wide range of beam energies [10]. The generator was used to produce a sample of central Au-Au collisions $(0-3.8 \mathrm{fm})$ at $\sqrt{s}=5 \mathrm{~A} \mathrm{GeV}$. The number of events was $5 \cdot 10^{5}$ for ${ }_{\Lambda}^{3} \mathrm{H}$ and $6.1 \cdot 10^{7}$ for ${ }_{\Lambda}^{4} \mathrm{H}$ and ${ }_{\Lambda}^{4} \mathrm{He}$ corresponding to respectively 30 minutes and 61 hours of NICA running time.

Particles produced by the event generators were transported through the detector using the GEANT3 transport package. The decay properties of hypernuclei (modes and branching ratios) have been introduced into GEANT from [11] (Tab. 1) and the lifetime has been taken to be the same as of $\Lambda$-hyperon.

\section{Analysis procedure}

The track reconstruction in MPD is based on the Kalman filtering method and the number of TPC points per track was required to be greater than 15 to ensure a good precision of 


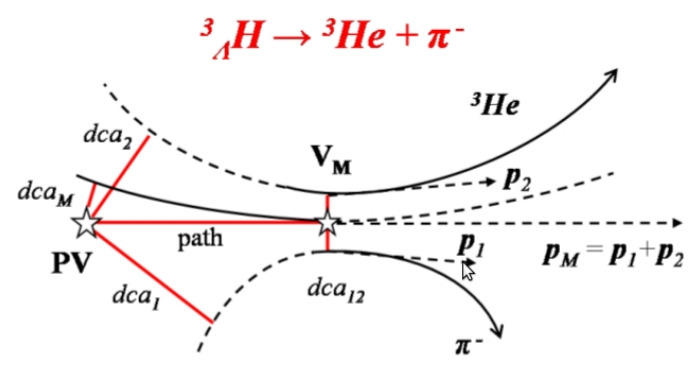

Figure 2. Event topology of two-particle decays of a charged particle (e.g. ${ }_{\Lambda}^{3} \mathrm{H} \rightarrow{ }^{3} \mathrm{He}+\pi^{-}$). Here $d c a_{1}$ and $d c a_{2}$ are the distances of the closest approach of the decay tracks to the primary vertex $P V$, $d c a_{12}$ is the distance between daughter tracks in the decay vertex $V_{M}, d c a_{M}$ is the distance of the closest approach of the mother particle to the primary vertex, path is the decay length, $p_{1}$ and $p_{2}$ are the momenta of daughter particles

Table 1. Hypernuclei decay modes and branching ratios under study

\begin{tabular}{llc}
\hline Particle & Decay chanel & Branching ratio \% \\
\hline${ }_{\Lambda}^{3} \mathrm{H}$ & $\pi^{-}+{ }^{3} \mathrm{He}$ & 24.7 \\
${ }^{3} \mathrm{H}$ & $\pi^{-}+\mathrm{p}+\mathrm{d}$ & 36.7 \\
${ }_{\Lambda}^{4} \mathrm{He}$ & $\pi^{-}+{ }^{3} \mathrm{He}+\mathrm{p}$ & 32.0 \\
${ }_{\Lambda}^{4} \mathrm{H}$ & $\pi^{-}+{ }^{4} \mathrm{He}$ & 75.0 \\
\hline
\end{tabular}

momentum and $d E / d x$ measurements. The signal event topology is shown in Fig. 2 along with the selection criteria: relatively large decay length of the mother particle, relatively large distance of the closest approach to the primary vertex of decay products, small track-to-track separation in the decay vertex. Both the DCA and two-track separation cuts should be more efficient if applied in $\chi^{2}$-space.

The exact values of the selection cuts were found by performing a multiple loops over the whole set of selection criteria with a requirement to maximize the invariant mass peak significance, defined as $S / \sqrt{S+B}$, where $S$ and $B$ are total numbers of signal (described by the Gaussian) and background (polynomial function) combinations inside $2 \sigma$ interval around the peak position.

\section{Results and discussions}

The results on hypernuclei reconstruction for species under study are presented in Figs. 3 and 4. Fig. 3 shows invariant mass distributions for ${ }_{\Lambda}^{4} \mathrm{H} \rightarrow{ }^{4} \mathrm{He}+\pi^{-}$and ${ }_{\Lambda}^{4} \mathrm{He} \rightarrow p+\pi^{-}+{ }^{3} \mathrm{He}$ decay modes. As mentioned above, the results have been obtained for $6.1 \cdot 10^{7}$ central events, corresponding to about 61 hours of NICA running time.

Fig. 4 shows invariant mass distributions for ${ }_{\Lambda}^{3} \mathrm{H}$ 2-prong and 3-prong decay modes. The results have been obtained for $5 \cdot 10^{5}$ central events, corresponding to about 30 minutes of NICA running time. The efficiency drop due to selection cuts comes from the necessity to suppress the combinatorial background in order to obtain a clean invariant mass peak, i.e. due to a larger combinatorial background stronger selection cuts were applied, which resulted in a lower efficiency. Based on the results of this study and model predictions, we have estimated the expected yield of particle species under interest for 10 weeks of data taking (see Tab. 2). 

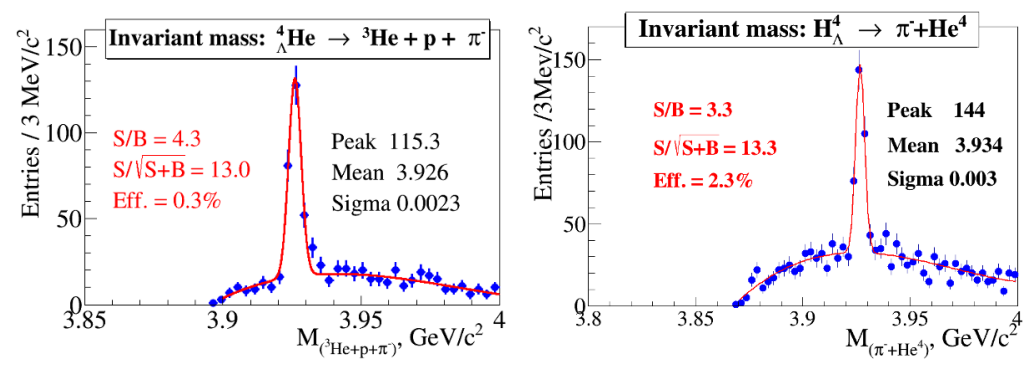

Figure 3. Left) reconstructed invariant mass of ${ }_{\Lambda}^{4} \mathrm{He} 3$-prong decay mode; right) reconstructed invariant mass of ${ }_{\Lambda}^{4} \mathrm{H}$ 2-prong decay mode
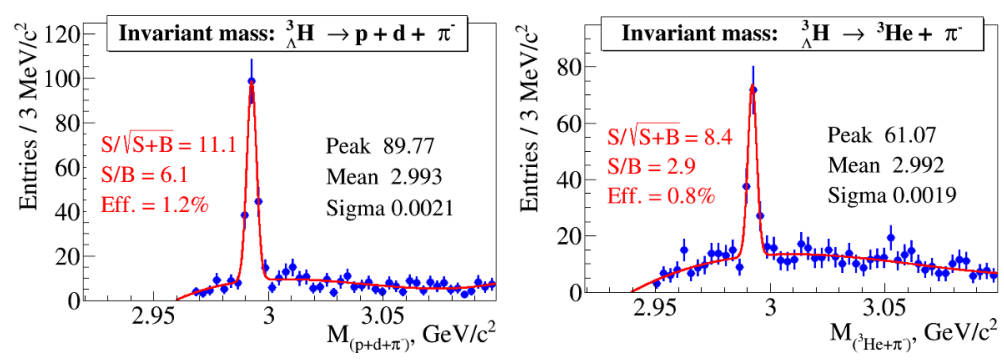

Figure 4. Left) reconstructed invariant mass of ${ }_{\Lambda}^{3} \mathrm{H}$ 3-prong decay mode; right) reconstructed invariant mass of 2-prong decay mode

Table 2. Expected particle yield for 10 weeks of NICA running time

\begin{tabular}{ll}
\hline Particle & Expected yield \\
\hline${ }_{\Lambda}^{3} \mathrm{H}$ & $8.1 \cdot 10^{5}$ \\
${ }_{\Lambda}^{4} \mathrm{He}$ & $1.4 \cdot 10^{5}$ \\
${ }_{\Lambda}^{4} \mathrm{H}$ & $1.9 \cdot 10^{5}$ \\
\hline
\end{tabular}

\section{Summary}

We have performed a simulation study of the MPD detector capabilities to reconstruct light hypernuclei in central Au+Au collisions at $\sqrt{s}=5 \mathrm{~A} \mathrm{GeV}$. The DCM-QGSM event generator was used to study the MPD detector Stage I set-up performance. The invariant mass resolution of $\approx 3 \mathrm{MeV} / c^{2}$ has been achieved. Based on the results of this study and model predictions, we have estimated the expected yield of particle species under interest for 10 weeks of data taking.

\section{References}

[1] http://nica.jinr.ru

[2] http://mpd.jinr.ru

[3] A.K. Kermann and M.S. Weiss, Phys. Rev. C 8, 408 (1973)

[4] A. Andronic et al., Phys. Lett. B 697, 203 (2011)

[5] V.D. Toneev and K.K. Gudima, Nucl. Phys. A 400, 173 (1983) 
[6] V.D. Toneev et al., Nucl. Phys. A 519, 463 (1990)

[7] N.S. Amelin et al., Sov. J. Nucl. Phys. 52, 272 (1990)

[8] T.A. Armstrong et al., Phys. Rev. Lett. 83, 5431 (1999)

[9] T.A. Armstrong et al., Phys. Rev. C 61, 064908 (2000)

[10] J. Steinheimer et al., Phys. Lett. B 714, 85 (2012)

[11] H. Kamada et al., Phys. Rev. C 57, 1595 (1998) 